\title{
Evaluation of Genetic and Environmental Parameters Determining Antibody Response Induced by Vaccination Against Foot and Mouth Disease
}

\author{
G. R. Gowane $\cdot$ A. K. Sharma $\cdot$ M. Sankar $\cdot$ P. Thirumurugan $\cdot$ \\ K. Narayanan $\cdot$ S. Subramaniam $\cdot$ B. Pattnaik
}

Received: 27 June 2012/ Accepted: 2 April 2013/Published online: 10 May 2013

(C) NAAS (National Academy of Agricultural Sciences) 2013

\begin{abstract}
Factors determining the variation in antibody response post foot and mouth disease virus (FMDV) vaccine were investigated in 395 sera samples of crossbred cattle maintained at the institute farm located in the temperate Himalayan region of India. Pre and post sera were tested using liquid phase blocking enzyme linked immunosorbent assay (LPBE) specific for serotypes $\mathrm{O}, \mathrm{A}$, and Asia1. All the pre-vaccination samples were unprotected and increase in the antibody titer was seen 28-day post-vaccination. All the samples were negative for infection status. Among the environmental determinants, cohort, season, and vaccination number proved to be significant sources of variation in the vaccine response $(P<0.05)$. Adult animals $(>1$ year $)$ were found to be significantly more protected than the younger animals $(P<0.05)$. Cows in the first trimester of lactation showed reduced protection by vaccine that might be attributed to the weakening of immune system consequent to the stress of production and calving as well as increased metabolic demands typical of a postpartum cow. The estimates of heritability $( \pm \mathrm{SE})$ for FMDV vaccine response for serotypes $\mathrm{O}$, A, and Asial were $0.17 \pm 0.07,0.03 \pm 0.06$, and $0.05 \pm 0.06$, respectively, indicating little scope of improvement after mass selection.
\end{abstract}

Keywords Vaccine $\cdot$ FMDV $\cdot$ Environment $\cdot$ Crossbred cattle $\cdot$ Genetics

\section{Introduction}

Foot and mouth disease virus (FMDV) represents one of the most devastating viruses affecting cloven-hoofed livestock including cattle, sheep, goats, and pigs. The disease is characterized by high fever and the formation of vesicles preferentially on tongue, lips, coronary band, teats, and udder, as well as between and above the claws of the feet.

G. R. Gowane $(\bowtie)$

Animal Genetics \& Breeding, Central Sheep \& Wool Research Institute, Via Jaipur, Avikanagar 304501, Rajasthan, India e-mail: gopalgowane@gmail.com

\footnotetext{
A. K. Sharma - M. Sankar · P. Thirumurugan · K. Narayanan Temperate Animal Husbandry Division, Indian Veterinary Research Institute, Nainital, Mukteshwar 263138, UK, India

S. Subramaniam - B. Pattnaik

Project Directorate on Foot and Mouth Disease, Nainital, Mukteshwar 263138, UK, India
}

The causative FMD virus is antigenically diverse having seven distinct serotypes and many variants within them. Being a single stranded RNA virus, it conforms to the quasispecies nature with emergence and reemergence of different genetic lineages with altered antigenicity within the serotypes, making vaccination-based control program prohibitively expensive, time consuming, and difficult to achieve [19]. FMDV is highly contagious, infection results in high morbidity and can lead to mortality in young stock. Therefore, the management and control of the disease is a major concern worldwide. The economic consequences of an FMD outbreak can be devastating as demonstrated by the 1997 FMD outbreak in Taiwan and the 2001 and 2007 outbreaks in the United Kingdom [7]. In India, during the period 2009-2010, a total of 799 outbreaks were reported as against 2962, 1467, 1211, and 511 outbreaks during 2005-2006, 2006-2007, 2007-2008, and 2008-2009, respectively [18]. Annual direct loss due to FMD in India has been estimated to the tune of Rs. 20,000 crores [19]. The threat of this virus is caused by a combination of 
virological characteristics including high contagiousness, virus tenacity, rapidity of replication, high level of virus dispersion via aerosols, and short incubation period [28]. FMD gains further significance due to its trans-boundary spread and trade sanctions imposed in countries where it occurs, affecting export of livestock and their products to countries free from this disease. In South Asia, FMD is endemic and predominantly associated with cattle and buffalo and currently FMD virus serotypes $\mathrm{O}, \mathrm{A}$, and Asia1 are prevalent. In FMD endemic regions such as Africa, Asia, and South America, control measures involve regular vaccination with inactivated virus vaccines; unfortunately, these vaccines have low efficacy and require regular boosts [1].

The high variability among animals in vaccine response, including significantly large numbers of non-responders is the new challenge, an example being the human recombinant hepatitis B vaccine [20]. There is considerable variation among animals in their response to infectious disease and vaccination, a significant proportion of which can be explained by host's genetics [5]. Considerable animal to animal variation has been reported in the context of FMDV challenge and immunisation with a variety of vaccine constructs including peptides $(1,10)$. However, the role of environmental determinants and host genetics as a function of variation to the response to immunisation has not been considered in much detail.

As cow slaughter is banned in India, vaccination remains the mainstay for the control of FMD. Therefore, this study was conceived with an objective to quantify the contribution as well as partition the impact of environmental and host genetic factors behind the variability in antibody response to inactivated trivalent $(\mathrm{O}, \mathrm{A}$, and Asia1) FMD vaccine in crossbred cattle. The advantage of the model that uses antibody response to FMDV as a phenotype is that animals can be immunized as per schedule, with absolutely no risk and under well-defined conditions.

\section{Materials and Methods}

Animals

The study population was a herd of crossbred cattle of Bosindicus and Bos-taurus, of which B. indicus constituted Hariana breed, whereas B. taurus constituted HolsteinFriesian (HF) alone or $\mathrm{HF}$ in combination with Jersey. Exotic inheritance level was more than $50 \%$. The herd was located at Indian Veterinary Research Institute (IVRI), Mukteswar (Nainital, Uttarakhand, India) in the temperate Himalayan region of India at $29^{\circ} 28^{\prime} \mathrm{N}, 79^{\circ} 38^{\prime} \mathrm{E}$, and has an average elevation of $2,171 \mathrm{~m}(7,123$ feet $)$ above the mean sea level (msl). Animals were raised separately according to their age group. The animals were maintained on a semi- intensive system with $5 \mathrm{~h}$ on pasture $(0800-1,300 \mathrm{~h})$ per day, standard ration and water ad lib. For newborn calves, milk was supplemented up to 3 months of age and from second week onwards green grass as well as calf starter feed was provided ad lib. The calf starter feed was continued up to 5 months. Calves were sent for grazing after they attained 3 months of age. The study used totally 395 samples over the period of two years (2010-2011).

\section{Vaccination and Sampling}

As part of the regular protective scheduled vaccination program, the animals were vaccinated ( $2 \mathrm{ml}$ intramuscular) with trivalent (O, A, and Asia1)-inactivated FMD vaccine adjuvanted with mineral oil (IVRI, Hebbal Bangalore, India). Whole blood was collected aseptically by jugular vein puncture from the crossbred cattle on " 0 " day (pre-vaccination) and 28-day post-vaccination (DPV) for serum separation. Serum was collected and stored at $-20{ }^{\circ} \mathrm{C}$ until testing.

Differentiation of Infected and Vaccinated Animals (DIVA)

The DIVA test developed in the PDFMD laboratory (http:// www.fao.org/ag/againfo/commissions/docs/training/manual/ SAARC_Training_Manual.pdf) was employed for the detection of infected animals from the vaccinated animals. Antibody against non structural proteins (NSP) $3 \mathrm{AB} 3$ is produced in FMDV-infected cattle (8-10 days after FMDV infection) but FMDV-free animals which have been vaccinated with inactivated purified vaccine do not produce antibody against NSP. In this test, anti-NSP (3AB3) antibody present in the serum sample is measured against purified recombinant $3 \mathrm{AB} 3$ antigen. OD was measured at the wavelength of $492 \mathrm{~nm}$ (reference $620 \mathrm{~nm}$ ). The results were interpreted based on the cut-off zones, such as if percent positivity (PP) value is more than $40 \%$ for $3 \mathrm{AB} 3$ NSP reactivity, then the sample was considered as positive. However, if PP value was less than $40 \%$ to $3 \mathrm{AB} 3 \mathrm{NSP}$ reactivity then the sample was considered as negative. All the animals were negative for DIVA indicating that the antibodies were produced in response to vaccination alone.

\section{LPBE for Detection of Antibody Against FMDV Vaccine}

Sera were tested by LPBE (PDFMD Mukteswar, India; http://www.fao.org/ag/againfo/commissions/docs/training/ manual/SAARC_Training_Manual.pdf) specific for serotypes O, A, and Asia1. Samples were tested in duplicate and plates were read in ELISA reader to obtain optical density (OD) values at $492 \mathrm{~nm}$ wavelength (reference $620 \mathrm{~nm}$ ). Calculated $\log _{10}$ values were recorded. Values 
more than 1.8 were protected and those less than 1.8 were unprotected. All the pre-vaccination samples were unprotective as its titer was below 1.8. Accordingly, there was no need for normalization of the 28 DPV titer data for prevaccination antibody level. The variability in the vaccineelicited immune response was assessed for all the three serotypes using Chi square test.

\section{Environmental Determinant Analysis}

The 28 DPV FMDV vaccination antibody titer obtained by LPBE was the phenotype for analyzing the variability in the immune response. Several factors such as cohort (year of birth), season of vaccination, sex of the animal, and number of vaccination were taken into consideration and their effect was analyzed using Logistic regression method on the dependent variable "antibody titer" (SPSS 16.0). In the logistic regression model, base for each level of factor was forced on the model. For cohort, base was year 2003. For vaccination number, base was one; for sex, base was female; and for season, base was post-monsoon (September-October). The statistical model used was as follows:

$$
\begin{aligned}
\operatorname{Ln}\left(P_{i} /\left(1-P_{i}\right)\right)= & \beta_{0}+\beta_{\mathrm{Y}} X_{\mathrm{Y}}+\beta_{\mathrm{S}} X_{\mathrm{S}}+\beta_{\mathrm{R}} X_{\mathrm{R}}+\beta_{\mathrm{V}} X_{\mathrm{Y}} \\
& +\varepsilon_{i j k l m}
\end{aligned}
$$

where $P_{i}=$ probability that animal $i$ born in year $j$ (jth cohort), vaccinated in the season $k$, of sex $l$ and vaccinated $m$ th time was protected by the vaccine against FMDV $(0=$ no protection (titer $<1.8$ ); $1=$ protection (titer $\geq 1.8$ )); $\beta_{0}=$ the intercept; $\beta_{\mathrm{Y}}=$ the regression coefficient for cohort effect; $\beta_{\mathrm{S}}=$ the regression coefficient for effect of season of birth; $\beta_{\mathrm{R}}=\mathrm{a}$ regression coefficient for effect of sex; $\beta_{\mathrm{V}}=\mathrm{a}$ regression coefficient for number of vaccination effect; $X_{\mathrm{Y}}$, $X_{\mathrm{S}}, X_{\mathrm{R}}, X_{\mathrm{V}}=$ the dummy variables for presentation of effects of cohort, season of birth, sex of the animal, and number of vaccination; and $\boldsymbol{\varepsilon}_{i j k l m}=$ random error term.

\section{Distribution of Protected Animals Across Different Age Groups}

The percent protected animals after inactivated trivalent FMDV vaccine were categorized according to age groups. Data on 395 observations was classified in four age groups: $<1$ year, $1-3$ years, $3-5$ years, and $>5$ years. Chi square test was used to check the significance of distribution for protected animals in different age groups.

Association of FMDV Vaccine-Elicited Immune Response with Milk Production Stress

A total of 88 animals were used for this study. The animals in the first trimester of lactation were categorized as in milk production stress, whereas those in later phase of lactation or non-lactating were considered free from milk-yield stress. Fisher's exact test was employed to see whether there is any association of milk production stress with vaccine response in cows in the first trimester of lactation versus non first trimester of lactation.

\section{Estimation of Heritability for FMDV Vaccine Response}

Responding variable was the FMDV vaccine-elicited immune response that was ordinal with five classes $(<1.5$, $1.68,1.98,2.28$, and $>2.4$ ), that were serially given $1-5$ codes and were used for genetic analysis. Genetic analysis was done by paternal half sib method using a derivativefree algorithm MTDFREML [2]. In the pedigree file, all the dams were given zero identity, and thus only sire and animal relationship were involved in numerator relationship matrix (NRM). Convergence of the REML solutions was assumed when the variance of function values $(-2 \log$ L) in the simplex was less than $10^{-8}$. To ensure that a global maximum is reached, analyses were restarted. When estimates did not change, convergence was confirmed. The single trait linear model constructed for estimating the genetic parameters was as follows:

$\mathbf{y}=\mathbf{X} \beta+\mathbf{Z}_{\mathbf{a}} \mathbf{a}+\varepsilon$

where $\mathbf{y}$ is the vector of records; $\boldsymbol{\beta}, \mathbf{a}$, and $\boldsymbol{\varepsilon}$ are vectors of fixed, direct additive genetic, and residual effects, respectively, with association matrices $\mathbf{X}$ and $\mathbf{Z}_{\mathbf{a}}$. Assumptions in the model were $\mathbf{V}(\mathrm{a})=\mathbf{A} \sigma_{\mathrm{a}}^{2}$ and $\mathbf{V}(\mathrm{e})=\mathbf{I} \sigma_{\mathrm{e}}^{2}$, where $\mathbf{I}$ is an identity matrix, $\mathbf{A}$ is the numerator relationship matrix between animals and $\sigma_{\mathrm{a}}^{2}$ and $\sigma_{\mathrm{e}}^{2}$ are additive direct and residual variances, respectively. Direct heritability was estimated using single trait analysis.

\section{Results and Discussion}

Variability Exists in the FMDV Vaccine-Elicited Immune Response

Sera samples of 28 DPV showed high antibody titer against all the three serotypes as compared to pre-vaccination samples (data not shown). We observed variation in the vaccineelicited immune response as some animals responded well, whereas others did not. Out of 395 sera samples screened for LPBE, only 33.16, 29.87, and $18.23 \%$ animals exhibited protective titer $\left(\log _{10}>1.8\right)$ against serotypes $\mathrm{O}, \mathrm{A}$, and Asia1, respectively. Differences between protected and unprotected animals were significant $(P<0.05)$ for all the vaccinations irrespective of serotypes (Table 1). Mammalian antibody responses to vaccination are complex polygenic traits modified by environment and host genetic factors 
Table 1 Number of protected and unprotected cattle against FMDV serotypes O, A, and Asia1

\begin{tabular}{|c|c|c|c|c|c|}
\hline Total samples & Date of vaccination & Status & $\mathrm{O}$ & A & Asia1 \\
\hline \multirow[t]{2}{*}{101} & \multirow[t]{2}{*}{ March 2010} & Protected & 29 & 6 & 18 \\
\hline & & Unprotected & 72 & 95 & 83 \\
\hline \multirow[t]{2}{*}{102} & \multirow[t]{2}{*}{ September 2010} & Protected & 33 & 54 & 25 \\
\hline & & Unprotected & 69 & 48 & 77 \\
\hline \multirow[t]{2}{*}{98} & \multirow[t]{2}{*}{ March 2011} & Protected & 46 & 38 & 25 \\
\hline & & Unprotected & 52 & 60 & 73 \\
\hline \multirow[t]{2}{*}{100} & October 2011 & Protected & 32 & 21 & 4 \\
\hline & & Unprotected & 68 & 79 & 96 \\
\hline$\chi^{2}$ Statistics & & & $P=0.00$ & $P=0.00$ & $P=0.00$ \\
\hline
\end{tabular}

Protected: $\log _{10}$ titer $\geq 1.8$; unprotected: $\log _{10}$ titer $<1.8$

[16]. There might be several genetic and non-genetic factors responsible for this variation. Several reports on vaccine response $[17,15,20,22,31,29,27,9,13,16]$ confirm the fact that vaccine response is variable in several populations. In the recent years, environmental and genetic factors have been shown to influence the vaccine-elicited immune response to FMD $[1,10]$.

\section{Environmental Determinant Analysis}

Estimates of Wald statistics and $\beta$ exponential for FMDV vaccine-elicited immune response $\left(\log _{10}<1.8=\right.$ unprotected; $\log _{10} \geq 1.8=$ protected) against serotypes $\mathrm{O}$, A, and Asial are presented in Table 2.

\section{Cohort}

The effect of cohort was significant $(P=0.001)$ on the vaccine-elicited immune response for serotype $O$. The base year differed significantly $(P \leq 0.05)$ from cohort of the years 2006, 2009, and 2010. It was observed that as the cohort level increased, the odds of protection by vaccine $(\beta$ exponential) also increased. Estimates of $\beta$ exponential were highest for years 2009 and 2010, indicating better protection level by the vaccine against serotype O. Effect of cohort was, however, found to be non-significant for serotypes A and Asia1 (Table 2). Nonetheless, for serotype A, it was observed that as the cohort level increased, the $\beta$ exponential also increased, indicating better performance of the later cohorts. The present finding is in agreement with the earlier report in Holstein-Charlois cattle, where significant effect of cohort on the antibody response to the FMDV vaccine was observed [10]. Similarly, significant effect of cohort on the antibody response to Bovine respiratory syncytial virus (BRSV) vaccination in the herd of HolsteinCharolais cattle was reported in the earlier study [16].

\section{Season}

Season of vaccination did not have any significant effect on the vaccine response $(P=0.253)$ for serotype $\mathrm{O}$ (Table 2$)$; however, season significantly influenced the vaccine-elicited immune response for serotypes A $(P=0.002)$ and Asia1 $(P=0.049)$. September/October (post-monsoon) vaccinated animals had better protection level as compared to that of March (spring or post-winter) vaccinated animals for serotype A (Table 2), whereas, March (spring or postwinter) vaccinated animals performed better for serotype Asial as compared to that of September/October (postmonsoon) vaccination (Table 2). This indicates that season of vaccination does not have any consistent determinant effect on the outcome of vaccine response.

\section{Sex}

Effect of sex of the animals on the vaccine-elicited immune response was found to be consistently non-significant $(P>0.05)$ for all the three serotypes (Table 2$)$. This finding is in conformity with the earlier report in which non-significant effect of sex was observed on antibody response to bovine herpesvirus1 vaccination [14]. Similarly, non-significant effect of sex on antibody response to the foreign antigens such as human erythrocytes and ovalbumin in dairy calves was observed [4]. In contrast to these reports, highly significant effect of sex on total BRSV-IgG level after BRSV vaccination on days 35 and 49 was observed [16]. BRSV-IgG in the female calves were 1.2-fold higher than in that of the male calves.

\section{Vaccination number}

Effect of number of vaccination on the FMDV vaccine response was highly significant $(P=0.00)$ for serotype $\mathrm{O}$, whereas it was significant for serotypes A $(P=0.011)$ and Asial $(P=0.048)$. It was observed that as the number of 
Table 2 Estimates of Wald statistics and $\beta$ exponential for FMDV vaccine-elicited immune response against serotypes O, A, and Asia1

\begin{tabular}{|c|c|c|c|c|c|c|c|c|c|}
\hline & \multicolumn{3}{|l|}{$\mathrm{O}$} & \multicolumn{3}{|l|}{ A } & \multicolumn{3}{|l|}{ Asia1 } \\
\hline & Wald & Significance & $\operatorname{Exp}(\beta)$ & Wald & Significance & $\operatorname{Exp}(\beta)$ & Wald & Significance & $\operatorname{Exp}(\beta)$ \\
\hline Cohort & 25.23 & 0.001 & & 12.766 & 0.120 & & 7.658 & 0.468 & \\
\hline 2004 & 3.03 & 0.082 & 3.25 & 0.847 & 0.357 & 1.74 & 1.625 & 0.202 & 2.98 \\
\hline 2005 & 1.88 & 0.170 & 2.98 & 0.013 & 0.910 & 0.92 & 1.859 & 0.173 & 3.80 \\
\hline 2006 & 5.87 & 0.015 & 9.26 & 1.029 & 0.310 & 2.40 & 1.843 & 0.175 & 4.83 \\
\hline 2007 & 1.34 & 0.246 & 3.39 & 0.678 & 0.410 & 2.20 & 0.003 & 0.954 & 1.08 \\
\hline 2008 & 1.94 & 0.163 & 4.96 & 0.019 & 0.889 & 1.17 & 0.325 & 0.569 & 2.28 \\
\hline 2009 & 9.70 & 0.002 & 59.68 & 2.646 & 0.104 & 7.28 & 0.910 & 0.340 & 4.39 \\
\hline 2010 & 9.12 & 0.003 & 66.38 & 1.913 & 0.167 & 6.16 & 0.637 & 0.425 & 3.64 \\
\hline 2011 & 3.05 & 0.081 & 23.79 & 0.000 & 0.999 & 0.00 & 0.000 & 0.999 & 0.00 \\
\hline Season (1) & 1.31 & 0.253 & 1.36 & 9.689 & 0.002 & 0.44 & 3.884 & 0.049 & 1.83 \\
\hline $\operatorname{Sex}(M)$ & 1.68 & 0.194 & 0.51 & 0.239 & 0.625 & 0.77 & 2.478 & 0.115 & 0.34 \\
\hline Vaccination Number & 30.02 & 0.000 & & 18.202 & 0.011 & & 14.164 & 0.048 & \\
\hline 2 & 12.89 & 0.000 & 7.76 & 6.404 & 0.011 & 4.39 & 2.226 & 0.136 & 2.47 \\
\hline 3 & 9.90 & 0.002 & 8.38 & 5.921 & 0.015 & 5.17 & 1.488 & 0.223 & 2.29 \\
\hline 4 & 11.84 & 0.001 & 19.19 & 2.951 & 0.086 & 4.04 & 0.241 & 0.623 & 1.49 \\
\hline 5 & 4.14 & 0.042 & 6.92 & 2.121 & 0.145 & 3.93 & 0.004 & 0.947 & 1.07 \\
\hline 6 & 5.96 & 0.015 & 12.53 & 1.563 & 0.211 & 3.76 & 0.448 & 0.503 & 0.45 \\
\hline 7 & 8.99 & 0.003 & 26.37 & 4.086 & 0.043 & 9.08 & 0.156 & 0.693 & 1.60 \\
\hline 8 & 1.38 & 0.241 & 3.99 & 0.058 & 0.810 & 1.33 & 1.524 & 0.217 & 0.18 \\
\hline Constant & 13.33 & 0.000 & 0.00 & 4.046 & 0.044 & 0.06 & 3.374 & 0.066 & 0.04 \\
\hline
\end{tabular}

Base: Cohort: 2003, season: 2, sex: F, Vaccination Number: 1

vaccination increased, the $\beta$ exponential for the outcome of protection against serotype $\mathrm{O}$ also increased; it was highest for vaccination numbers 4,6 , and 7 . Similarly for serotype A, vaccination number 7 showed highest odds of protection and for serotype Asia1, $\beta$ exponential was high for second, third, and seventh vaccination number. For all the three serotypes, we could see decline in the response after eighth vaccination. Repeated vaccination (till seventh) might have enhanced the memory cells for achieving higher response from the vaccine. In an earlier study, the older animals had significantly higher immune response for FMDV peptide vaccine, suggesting that even in older animals the immune system might still be developing [10]. In our study, reasons for decline in the response after eighth vaccination might represent plateau and saturation of antibody response and a subsequent decline can be construed as "immunological tachyphylaxis."

\section{Distribution of Protected Animals Across Different Age Groups}

The percent protected animals against serotypes $\mathrm{O}$, A, Asia1 in different age groups viz., $<1$ year, 1-3 years, $3-5$ years, and $>5$ years is presented in Table 3 . It was found that there was significant increase in percent protected animals from class 1 to class 3 for all the three serotypes $\mathrm{O}, \mathrm{A}$, and Asia1. However, there was decline in the number of protected animals for last category $(>5$ years) which included mostly lactating animals. The decline in vaccine-elicited immune response might be due to stressful physiological conditions especially in their first trimester of lactation.

In accordance with this, a study conducted on Israeli Friesian bulls [24] showed significant effect of age of the animals on the immune response to trivalent FMDV vaccine $\left(\mathrm{O}_{1}, \mathrm{~A}_{22}\right.$, and $\left.\mathrm{Asia}_{1}\right)$. Significant effect of age of the animals on antibody response to FMD vaccination in the Holstein-Charolais cattle was previously reported [10]. Calf age impacted the ability of a calf to mount an antibody response for Bovine viral diarrhea virus (BVDV) vaccine [6]; they reported that calves needed to be at least 130 days of age to elicit a positive response to vaccination. Significant effect of age on the vaccine-induced immune response on $\mathrm{IgG}_{1}$ and $\mathrm{IgG}_{2}$ levels after BRSV vaccination in the herd of Holstein-Charolais cattle was earlier reported [16]. Ageassociated responses have been described earlier for immunisation against antigens such as human serum albumin [11], keyhole limpet hemocyanin [21], BRSV, and Mycoplasma [30]. Earlier study [26] described that 6-month-old calves responded three times higher to vaccination against leptospiral bacterins than 3-month-old calves. 
Table 3 Percent protected crossbred cattle against serotypes O, A, Asia1 within different age groups

\begin{tabular}{llll}
\hline Age group (in years) & $\mathrm{O}$ & A & Asia1 \\
\hline$\leq 1$ & 25.00 & 13.46 & 11.54 \\
$1-3$ & 55.24 & 48.57 & 30.48 \\
$3-5$ & 41.33 & 30.67 & 22.67 \\
$>5$ & 17.79 & 22.70 & 10.43 \\
Overall & 33.16 & 29.87 & 18.23 \\
$\chi^{2}$ & $* *$ & $* *$ & $* *$ \\
\hline$* *$ Significance $<0.01$ & & &
\end{tabular}

$* *$ Significance $<0.01$

Table $4 \chi^{2}$ Test of significance between FMDV vaccine-elicited immune response and milk-yield stress

\begin{tabular}{|c|c|c|c|c|c|c|}
\hline \multirow[t]{2}{*}{ Classification of animals } & \multicolumn{2}{|l|}{$\mathrm{O}$} & \multicolumn{2}{|l|}{ A } & \multicolumn{2}{|c|}{ Asia1 } \\
\hline & ${ }^{\$} \mathrm{~T}$ & ${ }^{\#} \mathrm{~N}$ & ${ }_{\mathrm{T}} \mathrm{T}$ & ${ }^{\#} \mathrm{~N}$ & ${ }_{\mathrm{T}} \mathrm{T}$ & ${ }^{\#} \mathrm{~N}$ \\
\hline Protected & 4 & 41 & 3 & 40 & 1 & 49 \\
\hline Unprotected & 13 & 30 & 14 & 31 & 16 & 22 \\
\hline$\chi^{2}$ Test significance & \multicolumn{2}{|c|}{0.15} & \multicolumn{2}{|c|}{0.048} & \multicolumn{2}{|c|}{0.034} \\
\hline
\end{tabular}

$\$ T$ signifies number of animals in their first trimester of lactation

\# $N$ signifies animals in later part of lactation or non-lactating

\section{FMDV Vaccine-Elicited Immune Response} and Milk-Yield Stress

Cows in their first trimester of lactation did not elicit good protection against FMDV vaccine, whereas those in later phase of lactation as well as non-lactating were comparatively better protected (Table 4). Differences were, however, non-significant for serotype $\mathrm{O}$ and significant $(P<0.05)$ for serotypes A and Asia1. This might be due to the fact that lactating animals in their first trimester are under great physiological stress as they have to mobilize energy and protein resources for production state. To the best of our knowledge, this is the first study that showed negative influence of early stage of lactation on antibody response to FMDV vaccine that might be imputed to the stress of lactation. On the contrary, it has been shown that vaccination reduced the milk yield by $1.4 \mathrm{l} / \mathrm{cow}$ in case of bovine herpes virus [3].

\section{Genetic Parameters for FMDV Vaccine-Elicited Immune Response}

The estimates of heritability $( \pm \mathrm{SE})$ for serotypes $\mathrm{O}, \mathrm{A}$, and Asia1 were $0.17 \pm 0.070,0.03 \pm 0.056$, and $0.05 \pm 0.063$, respectively (Table 5). It was seen that the additive genetic variance for vaccine response was low in this herd. Majority of the phenotypic variance in the vaccine response was contributed by environmental variance $(\mathrm{Ve})$. The data that
Table 5 Genetic parameters for FMDV vaccine-elicited immune response in crossbred cattle

\begin{tabular}{llll}
\hline Parameters & Serotype O & Serotype A & Serotype Asia1 \\
\hline $\mathrm{Va}$ & 0.17864 & 0.03727 & 0.03973 \\
$\mathrm{Ve}$ & 0.86930 & 1.13486 & 0.75836 \\
$\mathrm{Vp}$ & 1.04794 & 1.17214 & 0.79809 \\
$h^{2}$ & $0.17 \pm 0.070$ & $0.03 \pm 0.056$ & $0.05 \pm 0.063$ \\
$e^{2}$ & $0.83 \pm 0.070$ & $0.97 \pm 0.056$ & $0.95 \pm 0.063$ \\
\hline
\end{tabular}

$V a$ genetic variance; $V e$ environmental variance; $V p$ phenotypic variance; $h^{2}$ heritability; $e^{2}$ environmental proportion of variance

were used for estimating the variance components and heritability were ordinal based on discrete categorization of antibody response on 28 DPV. There is usually an apprehension for use of ordinal or categorical data for estimating heritability as it is not continuous in nature. However, in this study, antibody response is a threshold trait that is always continuous genetically but dichotomous or ordinal phenotypically. Therefore, these data were used for genetic analysis considering its continuous internal nature being quantitative in genetic term. There has been considerable debate over the type of method to be used for evaluation of such traits. For most of the subjective scores in livestock, the best method of analyses remains controversial $[8,23$, 25]; however, accepting polygenic nature of these traits, genetic analyses of these traits is pertinent [12].

In this study, low estimate of additive genetic variance in the herd indicates little scope for improvement in the vaccine response through mass selection. Most of the variance in the vaccine response is explained by environmental proportion, and therefore, these factors need detailed exploration. In contrary to this study, significant effect of sire for IgG2 response to the FMDV 15 peptide and non-significant effect of sire for the $\mathrm{IgG} 1$ response was reported [10]. In another study, the contribution of sire was reported to be significant on post-vaccination days 14 and 35 for BRSV-IgG [16]. They reported total BRSV-IgG heritability $\left[h^{2}( \pm \mathrm{SE})\right]$ as $0.16( \pm 0.11)$ and $0.29( \pm 0.17)$ on days 14 and 35 , respectively.

\section{Conclusion}

Mammalian antibody responses are quantitative traits and are highly influenced by several environmental factors. This study elucidated the importance of environmental determinants such as cohort, repeated vaccination, age, and stage of lactation for significantly influencing the FMDV vaccine-elicited immune responses in crossbred cattle. Management being the first line of defense for controlling the disease in domestic animals needs more emphasis in all 
the livestock farms for obtaining better response. This study showed negative influence of early stage of lactation on antibody response to FMDV vaccine that might be ascribed to the stress of lactation as cows significantly change their priorities of the physiological needs. This study revealed little estimates of additive genetic variability for FMDV vaccine-elicited immune responses in cattle, indicating practically no scope of improvisation in vaccine response by mass selection. This information can be, however, utilized to progress toward the ultimate recognition of bovine genetic markers using genomic tools pertinent to optimal antibody production, as selection using DNA markers are independent of heritability estimates. This will lead to developing better strategies for protection against this dreaded disease.

Acknowledgments Authors are thankful to the Director IVRI for providing facilities and funds for carrying out the study. We duly acknowledge Director PDFMD for extending facilities to carry out LPBE and DIVA test. First author is grateful to Director CSWRI and Head AGB, CSWRI for their timely help.

Conflict of interest The authors declare that they have no conflict of interest.

\section{References}

1. Baxter R, Craigmile SC, Hailey C, Douglas AJ, Williams JL, Glass EJ (2010) BoLA-DR peptide binding pockets are fundamental for foot-and-mouth disease virus vaccine design in cattle. Vaccine 28:28-37

2. Boldman KG, Kriese LA, Van Vleck LD, Van Tussell CP, Kachman SD (1995) A manual for use of MTDFREML. A set of programs to obtain estimates of variances and covariances. ARS, USDA, Washington, DC

3. Bosch JC, Frankena K, van Oirschot JT (1997) Effect on milk production of vaccination with a bovine herpesvirus 1 genedeleted vaccine. Vet Rec 140:196-199

4. Burton JL, Burnside EB, Kennedy BW, Wilkie BN, Burton JH (1989) Antibody responses to human erythrocytes and ovalbumin as marker traits of disease resistance in dairy calves. J Dairy Sci 72:1252-1265

5. Davies G, Genini S, Bishop SC, Guiffra E (2009) An assessment of opportunities to dissect host genetic variation in resistance to infectious diseases in livestock. Animal 03:415-436

6. Downey ED, Rispath JF, Tait RG Jr, Garrick DJ, Reecy JM (2011) Environmental and managemental factors influencing BVDV antibody levels and response to vaccination in weaning calves. In: Proceedings 'Beef improvement federation' 43rd annual research symposium and annual meeting, Montana State University Bozeman, Montana, 1-4 June 2011, p 32

7. Grubman MJ, Moraes MP, Segundo FD, Pena L, Santos T (2008) Evading the host immune response: how foot-and-mouth disease virus has become an effective pathogen. FEMS Immunol Med Microbiol 53:8-17

8. Harvey WR (1982) Least squares analysis of discrete data. J Anim Sci 54:1067

9. Hohler T, Reuss E, Evers N, Dietrich E, Rittner C, Freitag CM, Vollmar J, Schneider P, Fimmers R (2002) Differential genetic determination of immune responsiveness to hepatitis B surface antigen and to hepatitis A virus: a vaccination study in twins. Lancet 360:991-995

10. Leach RJ, Craigmile SC, Knott SA, Williams JL, Glass EJ (2010) Quantitative trait loci for variation in immune response to a footand-mouth disease virus peptide. BMC Genet 11:107. doi: $10.1186 / 1471-2156-11-107$

11. Lie O (1979) Genetic analysis of some immunological traits in young bulls. Acta Vet Scand 20:372-386

12. Lourens A, Erasmus GJ, Schoeman SJ, van Wyk JB, Neser FWC, Steyn MG (1999) Evaluation of pelt traits in Karakul sheep applying linear and threshold models. S Afr J Anim Sci 29:11-14

13. Moreno CR, Lantier F, Berthon P, Gautier-Bouchardon AV, Boivin R, Lantier I, Brunel JC, Weisbecker JL, Francois D, Bouix J, Elsen JM (2003) Genetic parameters for resistance to the Salmonella abortusovis vaccinal strain Rv6 in sheep. Genet Select Evol 35:199-217

14. Muggli NE, Hohenboken WD, Cundiff LV, Mattson DE (1987) Inheritance and interaction of immune traits in beef calves. J Anim Sci 64:385-393

15. Newman MJ, Traux RE, French DD, Dietrich MA, Franke D, Stear MJ (1996) Evidence for genetic control of vaccine induced antibody responses in cattle. Vet Immunol Immunopathol 50:43-54

16. O'Neill RG, Woolliams JA, Glass EJ, Williams JL, Fitzpatrick JL (2006) Quantitative evaluation of genetic and environmental parameters determining antibody response induced by vaccination against bovine respiratory syncytial virus. Vaccine 24:4007-4016

17. Outteridge PM (1993) High and low responsiveness to vaccines in farm-animals. Immunol Cell Biol 71:355-366

18. Pattnaik B (2010) Annual report. Project Directorate on Foot \& Mouth Disease, Mukteswar

19. Pattnaik B, Subramaniam S, Sanyal A, Mohapatra JK, Dash BB, Ranjan R, Rout M (2012) Foot-and-mouth disease: global status and future road map for control and prevention in India. Agric Res 1(2):132-147

20. Poland GA, Jacobson RM (1998) The genetic basis for variation in antibody response to vaccines. Cur Opin Pediatrics 10:208-215

21. Pollock JM, Rowan TG, Dixon JB, Carter SD (1994) Level of nutrition and age at weaning: effects on humoral immunity in young calves. Br J Nutr 71:239-248

22. Raadsma HW, McEwan JC, Stear MJ, Crawford AM (1999) Genetic characterisation of protective vaccine responses in sheep using multi-valent Dichelobacter nodosus vaccines. Vet Immunol Immunopathol 72:219-229

23. Randall JH (1993) Some tools for the analysis of ordinal data. S Afr J Anim Sci 23:51

24. Samina I, Zakay-Rones Z, Weller JI, Peleg B (1998) Host factors affecting the homologous and heterologous immune response of cattle to FMDV: genetic background, age, virus strains and route of administration. Vaccine 16:335-339

25. Schoeman SJ, Albertyn JR (1993) An evaluation of the subjective categorization of hair quality pelt traits in Karakul lambs. S Afr J Anim Sci. 23:88

26. Scholium LM, Marshall RB (1985) Age and the ability of calves to respond to a leptospiral vaccine. N Z Vet J 33:146-147

27. Sitte K, Brinkworth R, East IJ, Jazwinska EC (2002) A single amino acid deletion in the antigen binding site of BoLADRB3 is predicted to affect peptide binding. Vet Immunol Immunopathol 85:129-135

28. Summerfield A, Guzylack-Piriou L, Harwood L, McCullough KC (2009) Innate immune responses against foot-and-mouth disease virus: current understanding and future directions. Vet Immunol Immunopathol 128:205-210

29. Tan PL, Jacobson RM, Poland GA, Jacobsen SJ, Pankratz VS (2001) Twin studies of immunogenicity-determining the genetic contribution to vaccine failure. Vaccine 19:2434-2439 
30. Thomas LH, Stott EJ, Howard CJ, Gourlay RN (1986) Development of a mutivalent vaccine against calf respiratory disease. In: Proceedings of the 14th World Buiatrics Congress, Dublin, pp 691-696
31. Wagter LC, Mallard BA, Wilkie BN, Leslie KE, Boettcher PJ, Dekkers JC (2000) A quantitative approach to classifying Holstein cows based on antibody responsiveness and its relationship to peripartum mastitis occurrence. J Dairy Sci 83:488-498 